\title{
Development of a probabilistic ocean modelling system based on NEMO 3.5: application at eddying resolution
}

\author{
Laurent Bessières $^{1}$, Stéphanie Leroux ${ }^{2}$, Jean-Michel Brankart ${ }^{2}$, Jean-Marc Molines ${ }^{2}$, Marie-Pierre Moine ${ }^{1}$, \\ Pierre-Antoine Bouttier ${ }^{2}$, Thierry Penduff ${ }^{2}$, Laurent Terray ${ }^{1}$, Bernard Barnier ${ }^{2}$, and Guillaume Sérazin ${ }^{1,2}$ \\ ${ }^{1}$ CNRS/CERFACS, CECI UMR 5318, Toulouse, France \\ ${ }^{2}$ Univ. Grenoble Alpes, CNRS, IRD, IGE, 38000 Grenoble, France \\ Correspondence to: Laurent Bessières (bessieres@cerfacs.fr)
}

Received: 5 July 2016 - Discussion started: 19 September 2016

Revised: 16 December 2016 - Accepted: 4 January 2017 - Published: 10 March 2017

\begin{abstract}
This paper presents the technical implementation of a new, probabilistic version of the NEMO ocean-sea-ice modelling system. Ensemble simulations with $N$ members running simultaneously within a single executable, and interacting mutually if needed, are made possible through an enhanced message-passing interface (MPI) strategy including a double parallelization in the spatial and ensemble dimensions. An example application is then given to illustrate the implementation, performances, and potential use of this novel probabilistic modelling tool. A large ensemble of 50 global ocean-sea-ice hindcasts has been performed over the period 1960-2015 at eddy-permitting resolution $\left(1 / 4^{\circ}\right)$ for the OCCIPUT (oceanic chaos - impacts, structure, predictability) project. This application aims to simultaneously simulate the intrinsic/chaotic and the atmospherically forced contributions to the ocean variability, from mesoscale turbulence to interannual-to-multidecadal timescales. Such an ensemble indeed provides a unique way to disentangle and study both contributions, as the forced variability may be estimated through the ensemble mean, and the intrinsic chaotic variability may be estimated through the ensemble spread.
\end{abstract}

\section{Introduction}

Probabilistic approaches, based on large ensemble simulations, have been helpful in many branches of Earth-system modelling sciences to tackle the difficulties inherent to the complex and chaotic nature of the dynamical systems at play. In oceanography, ensemble simulations have first been introduced for data assimilation purposes, in order to explic- itly simulate and, given observational data, reduce the uncertainties associated with, for example, model dynamics, numerical formulation, initial states, atmospheric forcing (e.g. Evensen, 1994; Lermusiaux, 2006). This type of probabilistic approach is also used to accurately assess ocean model simulations against observations (e.g. Candille and Talagrand, 2005), or to anticipate on the design of satellite observational missions (e.g. Ubelmann et al., 2009).

Performing ensemble simulations can be seen as a natural way to take into account the internal variability inherent to any chaotic and turbulent system, by sampling a range of possible trajectories of this system (independent and identically distributed). For example, long-term climate projections, or short-term weather forecasts, rely on large ensembles of atmosphere-ice-ocean coupled model simulations to simulate the probabilistic response of the climate system to various external forcing scenarios, or to perturbed initial conditions, respectively (e.g. Palmer, 2006; Kay et al., 2015; Deser et al., 2016)

The ocean is, like the atmosphere or the full climate system, a chaotic system governed by non-linear equations which couple various spatio-temporal scales. A consequence is that, in the turbulent regime (i.e. for $1 / 4^{\circ}$ or finer resolution), ocean models spontaneously generate a chaotic intrinsic variability under purely climatological atmospheric forcing, i.e. forced with the same repeated annual cycle from year to year. This purely intrinsic variability has a significant imprint on many ocean variables, especially in eddyactive regions, and develops on spatio-temporal scales ranging from mesoscale eddies up to the size of entire basins, and from weeks to multiple decades (Penduff et al., 2011; 
Grégorio et al., 2015; Sérazin et al., 2015). The evolution of this chaotic ocean variability under repeated climatological atmospheric forcing is sensitive to initial states. This suggests that turbulent oceanic hindcasts driven by the full range of atmospheric scales (e.g. atmospheric reanalyses) are likely to be sensitive to initial states as well, and their simulated variability should be interpreted as a combination of the atmospherically forced and the intrinsic/chaotic variability.

On the other hand, NEMO climatological simulations at $\sim 2^{\circ}$ resolution (in the laminar non-eddying regime) driven by a repeated climatological atmospheric forcing are almost devoid of intrinsic variability (Penduff et al., 2011; Grégorio et al., 2015). Because $\sim 1 / 4^{\circ}$-resolution OGCMs are now progressively replacing their laminar counterparts at $\sim 1-$ $2^{\circ}$ resolution used in previous CMIP-type long-term climate projections (e.g. HighResMIP, Haarsma et al., 2016), it becomes crucial to better understand and characterize the respective features of the intrinsic and atmospherically driven parts of the ocean variability, and their potential impact on climate-relevant indices.

Simulating, separating, and comparing these two components of the oceanic variability requires an ensemble of turbulent ocean hindcasts, driven by the same atmospheric forcing, and started from perturbed initial conditions. The high computational cost of performing such ensembles at global or basin scale explains why only a small number of studies have carried out this type of approach until now, and with small ensemble sizes (e.g. Combes and Lorenzo, 2007; Hirschi et al., 2013).

Building on the results obtained from climatological simulations, the ongoing OCCIPUT project (Penduff et al., 2014) aims to better characterize the chaotic low-frequency intrinsic variability (LFIV) of the ocean under a fully varying atmospheric forcing, from a large (50-member) ensemble of global ocean-sea-ice hindcasts at $1 / 4^{\circ}$ resolution over the last 56 years (1960-2015). The intrinsic and the atmospherically forced parts of the ocean variability are thus simulated simultaneously under a fully varying realistic atmosphere, and may be estimated from the ensemble spread and the ensemble mean, respectively. This strategy also allows investigation into the extent to which the full atmospheric variability may excite, modulate, damp, or pace intrinsic modes of oceanic variability that were identified from climatological simulations. OCCIPUT mainly focuses on the interannualto-decadal variability of ocean quantities having a potential impact on the climate system, such as sea surface temperature (SST), meridional overturning circulation (MOC), and upper ocean heat content (OHC).

This paper presents the technical implementation of the new, fully probabilistic version of the NEMO modelling system required for this project. It stands at the interface between scientific purposes and new technical developments implemented in the model. The OCCIPUT project is presented here as an application, to illustrate the system requirements and numerical performances. The mathematical background supporting our probabilistic approach is detailed in Sect. 2. Section 3 describes the new technical developments introduced in NEMO to simultaneously run multiple members from a single executable (allowing the online computation of ensemble statistics), with a flexible input-output strategy. Section 4 presents the implementation of this probabilistic model to perform regional and global $1 / 4^{\circ}$ ensembles, both performed in the context of OCCIPUT. The strategy chosen to trigger the growth of the ensemble spread, and the numerical performances of both implementations are also discussed. Section 5 finally presents some preliminary results from OCCIPUT to further illustrate potential scientific applications of this probabilistic approach. A summary and some concluding remarks are given in Sect. 6.

\section{From deterministic to probabilistic ocean modelling: mathematical background}

The classical, deterministic ocean model formulation can be written as follows:

$\mathrm{d} \boldsymbol{x}=\mathcal{M}(\boldsymbol{x}, t) \mathrm{d} t$,

where $\boldsymbol{x}=\left(x_{1}, x_{2}, \ldots, x_{N}\right)$ is the model state vector, $t$ is time, and $\mathcal{M}$ is the model operator, containing the expression of the tendency for every model state variable. An explicit time dependence is included in the model operator since the tendencies depend on the time-varying atmospheric forcing.

Computing a solution to Eq. (1) requires the specification of the initial condition at $t=0$, from which the future evolution of the system is fully determined. OCCIPUT investigates how perturbations in initial conditions evolve and finally affect the statistics of climate-relevant quantities. This problem may be addressed probabilistically by solving the Liouville equation:

$\frac{\partial p(\boldsymbol{x}, t)}{\partial t}=-\sum_{k=1}^{N} \frac{\partial}{\partial x_{k}}[\mathcal{M}(\boldsymbol{x}, t) p(\boldsymbol{x}, t)]$,

where $p(\boldsymbol{x}, t)$ is the probability distribution of the system state at time $t$. Equation (2) shows that this distribution is simply advected in the phase space by local model tendencies. In chaotic systems, small uncertainties in the initial condition $(p(\boldsymbol{x}, 0)$ concentrated in a very small region of the phase space) yield diverging trajectories; such systems are poorly predictable in the long term. In the turbulent ocean, the mesoscale turbulence and the LFIV have a chaotic behaviour. They both belong to what we will call, more generally, intrinsic variability in Sects. 4 and 5, in the sense that they do not result from the forcing variability but spontaneously emerge from the ocean even with constant or seasonal forcing. Because this intrinsic variability is chaotic, it can only be described in a statistical sense and the probabilistic approach of Eq. (2) is required. 


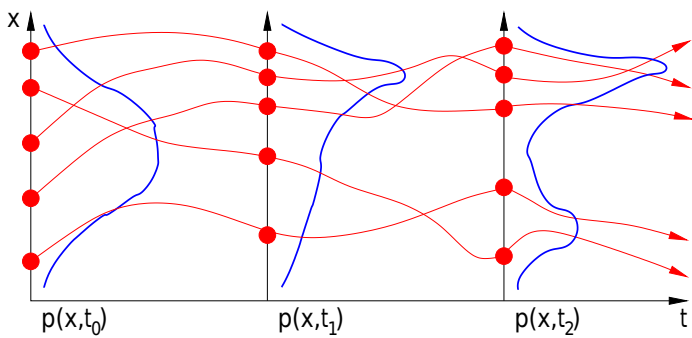

Figure 1. Schematic of an ensemble simulation (red trajectories), as an approximation to the simulation of an evolving probability distribution (in blue).

In addition to uncertainties in the initial condition, it is sometimes useful to assume that the model dynamics themselves are uncertain. This leads to a non-deterministic ocean model formulation, in which model uncertainties are described by stochastic processes. One possibility is, for instance, to modify Eq. (1) as follows.

$\mathrm{d} \boldsymbol{x}=\mathcal{M}(\boldsymbol{x}, t) \mathrm{d} t+\boldsymbol{\Sigma}(\boldsymbol{x}, t) \mathrm{d} \boldsymbol{W}_{t}$

In this equation, $\boldsymbol{W}_{t}$ is an $M$-dimensional standard Wiener process, and $\boldsymbol{\Sigma}(\boldsymbol{x}, t)$ is an $N \times M$ matrix, describing the influence of these processes on the model tendencies. Equation (3) does not include all possible ways of introducing a stochastic parameterization in a dynamical model, but it is sufficient to include the implementation that is described in this paper (in particular, to include the use of spacecorrelated or time-correlated autoregressive processes by expanding the definition of $\boldsymbol{x}$ in Eq. 3). With this additional stochastic term, Liouville equation transforms to FokkerPlanck equation:

$$
\begin{aligned}
\frac{\partial p(\boldsymbol{x}, t)}{\partial t}= & -\sum_{k=1}^{N} \frac{\partial}{\partial x_{k}}[\mathcal{M}(\boldsymbol{x}, t) p(\boldsymbol{x}, t)] \\
& +\frac{1}{2} \sum_{k=1}^{N} \sum_{l=1}^{N} \frac{\partial^{2}}{\partial x_{k} \partial x_{l}}\left[D_{\mathrm{kl}}(\boldsymbol{x}, t) p(\boldsymbol{x}, t)\right],
\end{aligned}
$$

where $D_{k l}(\boldsymbol{x}, t)=\sum_{p=1}^{M} \Sigma_{k p}(\boldsymbol{x}, t) \Sigma_{l p}(\boldsymbol{x}, t)$. The probability distribution $p(\boldsymbol{x}, t)$ is thus affected by the stochastic diffusion tensor $\mathbf{D}(\boldsymbol{x}, t)$ during its advection in the phase space by local model tendencies.

However, since Eqs. (2) and (4) are partial differential equations in an $N$ dimensional space, they generally cannot be solved explicitly for large size systems. Only an approximate description of $p(\boldsymbol{x}, t)$ can be obtained in most practical situations. A common solution is to reduce the description of $p(\boldsymbol{x}, t)$ to a moderate size sample, which can be viewed as a Monte Carlo approximation to Eqs. (2) and (4). This approach is illustrated in Fig. 1. The computation is initialized by a sample of the initial probability distribution $p\left(\boldsymbol{x}, t_{0}\right)$ (on the left in the figure), and each member of the sample is used as a different initial condition to Eqs. (1) and (3). The classical model operator can then be used to produce an ensemble of model simulations (red trajectories in the figures), which provide a sample of the probability distribution at any future time, e.g. $p\left(\boldsymbol{x}, t_{1}\right)$, or $p\left(\boldsymbol{x}, t_{2}\right)$.

This Monte Carlo approach is very general and can be also applied to any kind of stochastic parameterization (not only the particular case described by Eq. 3). It was first applied to ocean models in the framework of the ensemble Kalman filter (Evensen, 1994) to solve ocean data assimilation problems.

In summary, Eq. (1) describes the problem that is classically solved by the NEMO model; Eq. (3) is a modification of this problem with stochastic perturbations of the model equations that explicitly simulate model uncertainties; in this paper, this problem is solved using an ensemble simulation, which provides identically distributed realizations from the probability distribution, and thus a way to compute any statistic of interest.

\section{Performing ensemble simulations with NEMO}

The NEMO model (Nucleus for a European Model of the Ocean), described in Madec (2012), is used for oceanographic research, operational oceanography, seasonal forecasts and climate studies. This system embeds various model components (see http://www.nemo-ocean.eu/), including a circulation model (OPA, Océan PArallélisé), a sea-ice model (LIM, Louvain-la-Neuve ice model), and ecosystem models with various levels of complexity. Every NEMO component solves partial differential equations discretized on a 3-D grid using finite-difference approximations. The purpose of this section is to present the technical developments introduced in our probabilistic NEMO version, and to make the connection between these new developments and existing NEMO features.

\subsection{Ensemble NEMO parallelization}

The standard NEMO code is parallelized with MPI (message-passing interface) using a domain decomposition method. The model grid is divided in rectangular subdomains $(i=1, \ldots, n)$, so that the computations associated with each subdomain can be performed by a different processor of the computer. Spatial finite-difference operators require knowledge of the neighbouring grid points, so that the subdomains must overlap to allow the application of these operators on the discretized model field. Whenever needed, the overlapping regions of each subdomain must be updated using the computations made for the neighbouring subdomains. The NEMO code provides standard routines to perform this update. These routines use MPI to get the missing information from the other processors of the computer. This communication between processors makes the connection between subdomains in the model grid.

In practice, upon initialization one MPI communicator is defined with as many processors as subdomains, and each 


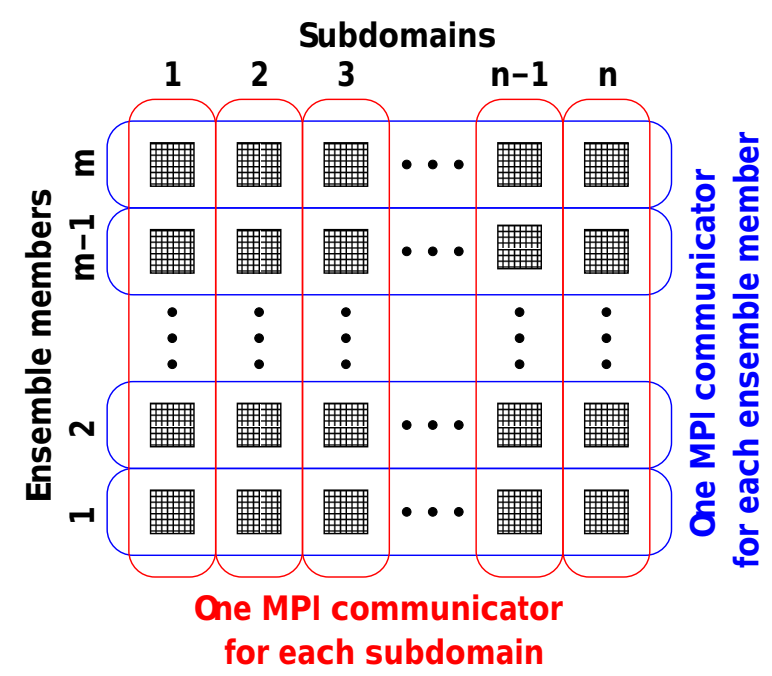

Figure 2. Schematic of the double parallelization introduced in NEMO: each processor (black squares) is dedicated to the computations associated with one model subdomain and one ensemble member. There is one MPI communicator within each ensemble member (in blue) to allow communications between neighbouring subdomains as in the standard NEMO parallelization, and there is one MPI communicator within each model subdomain (in red) to allow communication between ensemble members (e.g. to compute ensemble statistics online if needed). The total number of processors is thus equal to the product of the ensemble size by the number of subdomains $(m \times n)$.

processor is associated with a subdomain and knows which are its neighbours.

Ensemble simulations may be performed with NEMO by a direct generalization of the standard parallelization procedure described above. In other words, our ensemble simulations are performed from one single call to the NEMO executable, simply using more processors to run all members in parallel. This technical option is both natural and unnatural. It is natural since an ensemble simulation provides an approximate description of the probability distribution; it is thus conceptually appealing to advance all members together in time. It is unnatural since independent ensemble members may be run separately (in parallel, or successively) using independent calls to NEMO. However, the solution we propose is so straightforward that there is virtually no implementation cost, and is more flexible since the ensemble members may be run independently, by groups of any size, or all together. Furthermore, running all ensemble members together provides a new interesting capability: the characteristics of the probability distribution $p(\boldsymbol{x}, t)$ in Eq. (2) or (4) may be computed online, virtually at every time step of the ensemble simulation. This has been done using MPI to gather the required information from every member of the ensemble. These MPI communications make a natural connection between ensemble members, as a sample of the probability distribution $p(\boldsymbol{x}, t)$.
In practice, this implementation option only requires that at the beginning of the NEMO simulation, one MPI communicator is defined for each ensemble member, each one with as many processors as subdomains, so that each processor knows to which member it belongs, on which subdomain it is going to compute and what its neighbours are. Inside each of these communicators, each ensemble member may be run independently from the other members, without changing anything else in the NEMO code. However, all members are obviously not supposed to behave exactly the same: the index of the ensemble member must have some influence on the simulation. This influence may be in the name of the files defining the initial condition, parameters or forcing, or in the seeding of the random number generator (if a random forcing is applied, as in Eq. 3). The index of the ensemble member must also be used to modify the name of all output files, so that the output of different members is saved in different files. As it appears, this implementation of ensemble simulations does not require much coding effort (a few tens of lines in NEMO, partly because most of the basic material was already available in the original code). More technical details about this can be found in Sect. 7 .

In summary, the NEMO ensemble system relies on a double parallelization, over model subdomains and over ensemble members, as illustrated in Fig. 2. In this algorithm, ensemble simulations are thus intricately linked to MPI parallelization. There is no explicit loop over the ensemble members; this loop is done implicitly through MPI; running more ensemble members means either using more processors or using less processors for each member.

\subsection{Online ensemble diagnostics}

As mentioned above, one important novelty offered by the ensemble NEMO parallelization is the ability to compute online any feature of the probability distribution $p(\boldsymbol{x}, t)$. This can be done within additional MPI communicators connecting all ensemble members for each model subdomain (in red in Fig. 2). MPI sums in these communicators are for instance immediately sufficient to estimate the following:

- the mean of the distribution:

$$
\begin{aligned}
\mu_{k} & =\int x_{k} p(\boldsymbol{x}, t) \mathrm{d} \boldsymbol{x} \\
\sim \widetilde{\mu}_{k} & =\frac{1}{m} \sum_{j=1}^{m} x_{k}^{(j)},
\end{aligned}
$$

where $x_{k}$ is one of the model state variables, $x_{k}^{(j)}$ is this variable simulated in member $j, \mu_{k}$ is the mean of the distribution for this variable, and $\widetilde{\mu}_{k}$ is the estimate of the mean obtained from the ensemble. It is interesting to note that the sum over ensemble members in Eq. (5) is not explicitly coded in NEMO; it is performed instead by a single call to MPI, which computes the sums over 
all processors of the ensemble communicators (in red in Fig. 2). The same remark also applies to the sums in the following equations.

- the variance of the distribution:

$$
\begin{gathered}
\sigma_{k}^{2}=\int\left(x_{k}-\mu_{k}\right)^{2} p(\boldsymbol{x}, t) \mathrm{d} \boldsymbol{x} \\
\sim \widetilde{\sigma}_{k}^{2}=\frac{1}{m-1} \sum_{j=1}^{m}\left(x_{k}^{(j)}-\widetilde{\mu}_{k}\right)^{2},
\end{gathered}
$$

where $\sigma_{k}^{2}$ is the variance of the distribution for variable $x_{k}$ and $\widetilde{\sigma}_{k}^{2}$ is the estimate obtained from the ensemble. The ensemble standard deviation is simply the square root of $\widetilde{\sigma}_{k}^{2}$.

- Ensemble covariance between two variables at the same model grid point:

$$
\begin{gathered}
\gamma_{k l}=\int\left(x_{k}-\mu_{k}\right)\left(x_{l}-\mu_{l}\right) p(\boldsymbol{x}, t) \mathrm{d} \boldsymbol{x} \\
\sim \tilde{\gamma}_{k l}=\frac{1}{m-1} \sum_{j=1}^{m}\left(x_{k}^{(j)}-\tilde{\mu}_{k}\right)\left(x_{l}^{(j)}-\tilde{\mu}_{l}\right),
\end{gathered}
$$

where $\gamma_{k l}$ is the covariance between variables $x_{k}$ and $x_{l}$, and $\widetilde{\gamma}_{k l}$ is the estimate obtained from the ensemble.

This is directly generalizable to the computation of higherorder moments (skewness, kurtosis), which is then reduced to MPI sums in the ensemble communicators. Moreover, simple MPI algorithms can also be designed to compute many other probabilistic diagnostics online, such as the rank of each member in the ensemble, and from there, estimates of quantiles of the probability distribution. Specific applications of this feature are discussed in Sect. 5.

This online estimation of the probability distribution, via the computation of ensemble statistics, opens another interesting new capability: the solution of the model equations may now depend on ensemble statistics, available at each time step if needed. For instance, it may be interesting to relax the modelled forced variability towards reference (e.g. reanalysed or climatological) fields, with no explicit damping of the intrinsic variability: the nudging term would involve the current ensemble mean and be applied identically to all members at the next time step, resulting in a simple "translation" of the entire ensemble distribution toward the reference field.

Other applications, such as ensemble data assimilation, may also require an online control of the ensemble spread, which is hereby made possible within NEMO.

\subsection{Connection with NEMO stochastic parameterizations}

Ensemble simulations are directly connected to stochastic parameterizations (as introduced in Eq. 3). In NEMO, stochastic parameterizations have recently been implemented to explicitly simulate the effect of uncertainties in the model (Brankart et al., 2015). In practice, this is done by generating maps of autoregressive processes, which can be used to introduce perturbations in any component of the model. In Brankart et al. (2015), examples are provided to illustrate the effect of these perturbations in the circulation model, in the ecosystem model and in the sea ice model. For instance, a stochastic parameterization was introduced in the circulation model to simulate the effect of unresolved scales in the computation of the large-scale density gradient, as a result of the nonlinearity of the sea water equation of state (Brankart, 2013). This particular stochastic parameterization is switched on during one year in order to initiate the dispersion of the OCCIPUT ensemble simulations started from a single initial condition (see Sect. 4).

\subsection{Connection with NEMO data assimilation systems}

Ensemble model simulations are also key in ensemble data assimilation systems: they propagate, in time, uncertainties in the model initial condition, and provide a description of model uncertainties in the assimilation system (e.g. using stochastic perturbations). Data assimilation can then be carried out by conditioning this probability distribution to the observations whenever they are available. The ensemble data assimilation method that is currently most commonly used in ocean applications is the Ensemble Kalman filter (Evensen, 1994), which performs the observational update of the model probability distributions with the assumption that they are Gaussian. However, it has been recently suggested that the Gaussian assumption is often insufficient to correctly describe ocean probability distributions, and that more general methods using, for instance, anamorphosis transformations (Bertino et al., 2003; Brankart et al., 2012) or a particle filtering approach (e.g. Van Leeuwen, 2009) may be needed. One of the purposes of the SANGOMA European project is precisely to develop such more general methods for ocean applications, and to implement them within NEMO-based ocean data assimilation systems (e.g. Candille et al., 2015). In these methods, the role of ensemble NEMO simulations is even more important since they require a more detailed description of the probability distributions (compared to the Gaussian assumption, which only requires the mean and the covariance). The importance of ensemble simulations in data assimilation certainly explains why the ensemble NEMO parallelization (introduced above) has been first applied within SANGOMA, to assimilate altimetric observations in an eddying NEMO configuration of the North Atlantic (Candille et al., 2015). 


\subsection{Connection with the NEMO observation operator and model assessment metrics}

Another important benefit of the probabilistic approach is to consolidate and objectivate statistical comparisons between actual observations and model-derived ensemble synthetic observations. Probabilistic assessment metrics are commonly used in the atmospheric community (e.g. Toth et al., 2003) but are quite new in oceanography. Briefly speaking, these methods generally quantify two attributes of an ensemble simulation: the reliability and the resolution. An ensemble is reliable if the simulated probabilities are statistically consistent with the observed frequencies. The ensemble resolution is related to the system ability to discriminate between distinct observed situations. If the ensemble is reliable, the resolution is directly related to the information content (or the spread) of the probability distribution. A popular measure of these two attributes is, for instance, provided by the continuous rank probability score (CRPS), which is based on the square difference between a cumulative distribution function (CDF) as provided by the ensemble simulation and the corresponding CDF of the observations (Candille and Talagrand, 2005).

In OCCIPUT, such probabilistic scores will be computed from real observations and from the ensemble synthetic observations (along-track Jason-2 altimeter data and ENACTENSEMBLES temperature and salinity profile data) generated online using the existing NEMO observation operator (NEMO-OBS module). NEMO-OBS is used exactly as in standard NEMO within each member of the ensemble, thereby providing an ensemble of model equivalents for each observation rather than a single value. Probabilistic metrics (i.e. CRPS score) will then be computed to assess the reliability and resolution of the OCCIPUT simulations.

\subsection{Connection with NEMO I/O strategy}

Our implementation of ensemble NEMO using enhanced parallelization is technically not independent from the NEMO I/O strategy. Indeed, in NEMO, the input and output of data is managed by an external server (XIOS, for XML IO Server), which is run on a set of additional processors (not used by NEMO). The behaviour of this server is controlled by an XML file, which governs the interaction between XIOS and NEMO, and which defines the characteristics of input and output data: model fields, domains, grid, I/O frequencies, time averaging for outputs, etc. To exchange data with disk files, every NEMO processor makes a request to the XIOS servers, consistently with the definitions included in the XML file. In this operation, the XIOS servers buffer data in memory, with the decisive advantage of not interrupting NEMO computations with the reading or writing in disk files. One peculiarity of this buffering is that each XIOS server reads and writes one stripe of the global model domain (along the second model dimension), and thus exchanges data with processors corresponding to several model subdomains. To optimize the system, it is obviously important that the number of XIOS servers (and thus the size of these stripes) be correctly dimensioned according to the amount of I/O data, which may heavily depend on the model configuration and on the definition of the model outputs.

To use XIOS with our implementation of ensemble NEMO for OCCIPUT, we thus had to take care of the two following issues. First, different ensemble members must write different files. This problem could be solved because XIOS was already designed to work with a coupled model, and can thus deal with multiple contexts: i.e. one for each of the coupled model components. It was thus directly possible to define one context for each ensemble member, just as if they were different components of a coupled model. Second, in ensemble simulations, the amount of output data is proportional to the ensemble size, so that the number of XIOS servers must be increased accordingly, albeit with some care, because the size of the data stripe that is processed by each server should not be reduced too much.

\section{Example of application: the OCCIPUT project}

The implementation of this ensemble configuration of NEMO was motivated to a large extent by the scientific objectives of the OCCIPUT project, described in the introduction. In this section, we present two ensemble simulations, E-NATL025 and E-ORCA025, performed in the context of this project. We focus on the model set-up, the integration strategy, and the numerical performances of the system, followed by a few illustrative preliminary results in Sect. 5 .

\subsection{Regional and global configurations}

E-ORCA025 is the main ensemble simulation aimed for OCCIPUT. It is a 50-member ensemble of global ocean-sea-ice hindcasts at $1 / 4^{\circ}$ horizontal resolution, run for 56 years. Before performing this large ensemble, a smaller (20-year $\times 10$ member) regional ensemble simulation, E-NATL025, was performed on the North Atlantic domain in order to test the new system implementation and to validate the stochastic perturbation strategy for triggering the growth of the ensemble dispersion. The global and the regional ensemble configurations are both based on version 3.5 of NEMO, and use a $1 / 4^{\circ}$ eddy-permitting quasi-isotropic horizontal grid ( $\sim 27 \mathrm{~km}$ at the equator), the grid size decreasing poleward. Table 1 summarizes the characteristics of ensembles. The model parameters are very close to those used in the DRAKKAR-ORCA025 ${ }^{1}$ one-member setups (Barnier et al., 2006), the present setup using a greater number (75) of vertical levels (see Table 1). They are also close to those used for the 327-year ORCA025-MJM01 one-member climatologi-

\footnotetext{
${ }^{1}$ DRAKKAR-ORCA025 website: http://www.drakkar-ocean. eu/global-models/orca025
} 
cal simulation used in Penduff et al. (2011), Grégorio et al. (2015), and Sérazin et al. (2015) to study various imprints of the LFIV under seasonal atmospheric forcing.

\subsection{Integration and stochastic perturbation strategies}

A one-member spin-up simulation is first performed for each ensemble. For the regional ensemble (E-NATL025), it is performed from 1973 (cold start) to 1992, forced with DFS.5.2 atmospheric conditions (Dussin et al., 2016). For the global ensemble (E-ORCA025), the spin-up strategy has to be adapted to match the OCCIPUT objective to perform the ensemble hindcast over the longest period available in the atmospheric forcing DFS5.2 (i.e. 1960-2015). The onemember spin-up simulation is thus performed as follows: (1) it is first forced by the standard DFS5.2 atmospheric forcing from 1 January 1958 (cold start) to 31 December 1976; (2) this simulation is continued over January 1977 with a modified forcing function that linearly interpolates between 1 January 1977 and 31 January 1958; (3) the standard DFS5.2 forcing is applied again normally from 1 February 1958 to the end of 1959. This 21-year spin-up (19581977, then 1958-1959) thus includes a smooth artificial transition from January 1977 back to January 1958. This choice was made as a compromise to maximize the duration of the single-member spin-up simulation and of the subsequent ensemble hindcast, while minimizing the perturbation in the forcing during the transition, since 1977 was found to be a reasonable analogue of 1958 in terms of key climate indices (El Niño Southern Oscillation, North Atlantic Oscillation, and Southern Annular Mode).

The $N$ members of both ensemble simulations (i.e. $N=$ 10 for E-NATL025 and $N=50$ for E-ORCA025) are started at the end of the single-member spin-up; a weak stochastic perturbation in the density equation, as described by Eq. (3) and in Sect. 3.3 (see also Brankart et al., 2015) is then activated within each member. This stochastic perturbation is only applied for 1 year to seed the ensemble dispersion (during 1993 for E-NATL025, during 1960 for E-ORCA025). It is then switched off throughout the rest of the ensemble simulations. Once the stochastic perturbation is stopped, the $N$ members are thus integrated from slightly perturbed initial conditions (i.e. 19 more years for E-NATL025 and 55 more years for E-ORCA025), but forced by the exact same atmospheric conditions (DFS5.2, Dussin et al., 2016). The code is parallelized with the double-parallelization technique described in Sect. 3.1 so that the $N$ members are integrated simultaneously through one single executable.

\subsection{Performance of the NEMO ensemble system in OCCIPUT configurations}

The regional ensemble (E-NATL025) was performed to test the system implementation and to calibrate the global configuration. The global ensemble simulation E-
ORCA025 represents, in total, 2821 cumulated years of simulation (56 years $\times 50$ members +21 years of onemember spin-up) over 110 million grid points (longitude $\times$ latitude $\times$ depth $=1442 \times 1021 \times 75$ ). As confirmed thereafter in Fig. 3, integrating such a system within one executable with reasonable wall-clock time, and managing its outputs lies beyond national or regional European centres computational capabilities (i.e. Tier-1 systems), requiring systems that can provide European top capabilities, which are beyond the Petaflops level (i.e. Tier- 0 systems).

All simulations were performed between 2014 and 2016 on the French Tier- 0 Curie supercomputer, supported by PRACE (Partnership for Advanced Computing in Europe) and GENCI (Grand Equipement National de Calcul Intensif, French representative in PRACE) grants $\left(19.10^{6} \mathrm{HCPU}\right.$, see details below). Curie is a Bull system (BullX series designed for extreme computing) based on Intel processors. The architecture used for the simulations is the one of the "Curie thin nodes" configuration (Curie-TN), which is mainly targeted at MPI parallel codes and includes more than 80000 Intel Sandy Bridge computing cores (Peak frequency per core: $2.7 \mathrm{GHz}$ ) gathered in 16-core nodes of $64 \mathrm{~GB}$ of memory.

Preliminary tests showed that the one-member ORCA025 configuration has a good scalability up to 400 cores on CurieTN (not shown). In order to test the ensemble global configuration on Curie-TN, short 180-step experiments were run, disregarding the first and last steps (which correspond to reading and writing steps, respectively, that are performed only once during production jobs). The performance of the system was measured in steps per minute by analysing the 160 steps in between (steps 10 to 170). Figure 3a shows this measure of the system performance (in steps $\mathrm{min}^{-1}$ ) as a function of the number of members, for different domain decompositions $\left(64,128,160,256\right.$, and 400 cores member $\left.{ }^{-1}\right)$. It appears that the performance is independent of the ensemble size for domain decomposition up to 160 domains per member. When more than 160 domains per member are used, the performance starts to decrease for increasing ensemble size, from 25 members (10) for the decomposition with 256 (400) domains per member. Fluctuations in steps per minute may appear (see the performance for the decomposition with 400 domains per member and 25 members in Fig. 3a), depending on machine load and file system stability (the performance of this specific point has not been reassessed for CPU cost reasons). The scalability of the global ensemble configuration E-ORCA025, as aimed for in OCCIPUT $(N=50)$, is shown in Fig. 3b: the efficiency is measured as the ratio of the observed speedup to the theoretical speedup, relative to the smallest domain decomposition tested, i.e. with 3200 cores $(50 \times 64)$. The efficiency is remarkably good and remains around $90 \%$ for 20000 used cores.

Based on these performance tests, a domain decomposition with relatively few cores was chosen in order to maintain a manageable rate of I/Os. The decomposition with 128 cores per member has been retained (corresponding to the 
Table 1. Main characteristics of the NEMO 3.5 set-up used for the regional and global OCCIPUT ensembles.

\begin{tabular}{lll}
\hline & Regional ensemble & Global ensemble \\
\hline Ensemble name & E-NATL025 & E-ORCA025 \\
Spatial domain & North Atlantic $\left(21^{\circ} \mathrm{S}-81^{\circ} \mathrm{N}\right)$ & Global \\
Horizontal resolution & $1 / 4^{\circ}(486 \times 530$ grid points $)$ & $1 / 4^{\circ}(1441 \times 1021$ grid points $)$ \\
Vertical resolution & 46 levels & 75 levels \\
\hline Ensemble size & 10 members & 50 members \\
Time periods covered by ensemble & $1993-2012$ & $1960-2015$ \\
Stochastic perturbation phase & 1 year $(1993)$ & 1 year $(1960)$ \\
\hline Surface boundary conditions & DFS5.2 (Dussin et al., 2016) & \\
& Turbulent air-sea fluxes bulk formula \\
& (with absolute wind) & \\
& SSS relaxation in each member 50 m/(300 days) piston velocity \\
\hline
\end{tabular}

red line in Fig. 3) so that $50 \times 128=6400$ cores are used for the ensemble NEMO system.

In order to optimize and to make the I/O data flux management flexible, 40 XIOS servers have been run as independent MPI tasks in detached mode, allowing the overlap of I/O operations with computations. Compared to the 10member regional case, the 50-member global case required a larger XIOS buffer size. For this reason, each of the 40 $\mathrm{XIOS}$ instances was run on a dedicated and exclusive Curie$\mathrm{TN}$, allowing each server to use the entire memory available on each 16 -core node (i.e. $64 \mathrm{~GB}$ ); the 40 XIOS servers thus used $16 \times 40=640$ cores in total. The integration of the 50 member global E-ORCA025 ensemble therefore required the use of $6400+(40 \times 16)=7040$ cores.

XIOS makes use of parallel file system capabilities via the Netcdf4-HDF5 format, which allows both online data compression and parallel I/O. Therefore, XIOS is used in "multiple file" mode where each XIOS instance writes a file for one stripe of the global domain, yielding 40 files times 50 members for each variable and each time. At the end of each job, the 40 stripes are recombined on-the-fly into global files.

Preliminary tests have shown that the 50-member E-ORCA025 global configuration performs about 20 steps $\min ^{-1}$, including actual $\mathrm{I} / \mathrm{O}$ fluxes and additional operations (e.g. production of ensemble synthetic observations). Since the numerical stability of this global setup requires a model time step of $720 \mathrm{~s}$, about 2 million time steps, 85 days of elapse time, and about 14.4 million core hours were needed in theory to perform the 56-year OCCIPUT ensemble simulation. The final CPU cost of the global ensemble experiment was about 19 million CPU hours, due to fluctuations in model efficiency, occasional problems on file systems which required the repetition of certain jobs, the need to decrease the model time step (increased high-frequency variance in the wind forcing data over the last decades), and the online computation of ensemble diagnostics (high-frequency ensemble covariances, all terms of the heat content budget ensemble) over the last decade. The cost of online ensemble diagnostics depends on the call frequency, number, and size of the concerned fields, on the architecture of the machine, and on the performance of communications. Our online ensemble diagnostics concerned a few two-dimensional fields at hourly to monthly frequencies, and had a negligible cost.

The final E-ORCA025 global database is saved in Netcdf4-HDF5 format (chunked and compressed, compression ratio in italics below). The primary dataset produced by the model consists of the following: monthly averages for fully 3 -D fields (56 years $\times 12$ months $\times 50$ members $\times 2.8 \mathrm{~GB} \times 41.5 \%=39 \mathrm{~TB}), 5$-day averages for 162 $D$ fields ( 56 years $\times 50$ members $\times 6.8 \mathrm{~GB} \times 30 \%=6 \mathrm{~TB}$ ), the Jason-2 and ENACT-ENSEMBLES ensemble synthetic observations (5 TB), and hourly ensemble statistics for key variables (1 TB). One restart file per member and per year is also archived (about $35 \mathrm{~TB}$ after compression). We then computed a secondary dataset, consisting in 50-member yearly/decadal averages of the 3-D-fields (2TB), ensemble deciles of monthly/yearly/decadal 3-D-fields (6 TB), and data associated with on-line monitoring (1 TB). The total output amounts to less than $100 \mathrm{~TB}$ and 100000 inodes on the Curie-TN file system.

\section{Preliminary results from the OCCIPUT application}

We now present some preliminary results from the regional and global OCCIPUT ensemble simulations described in Sect. 4.1 , in order to illustrate the concepts and the technical implementation presented above.

\subsection{Probabilistic interpretation}

Figure 4 shows, for the 10-member regional ensemble, the 1993-2012 time series of monthly temperature anomalies at depth $93 \mathrm{~m}$ at two contrasting grid points: in the Gulf Stream and in the middle of the North Atlantic subtropical gyre. Figure $4 \mathrm{a}$ and $\mathrm{c}$ represent a sample of $N$ trajectories of the tem- 

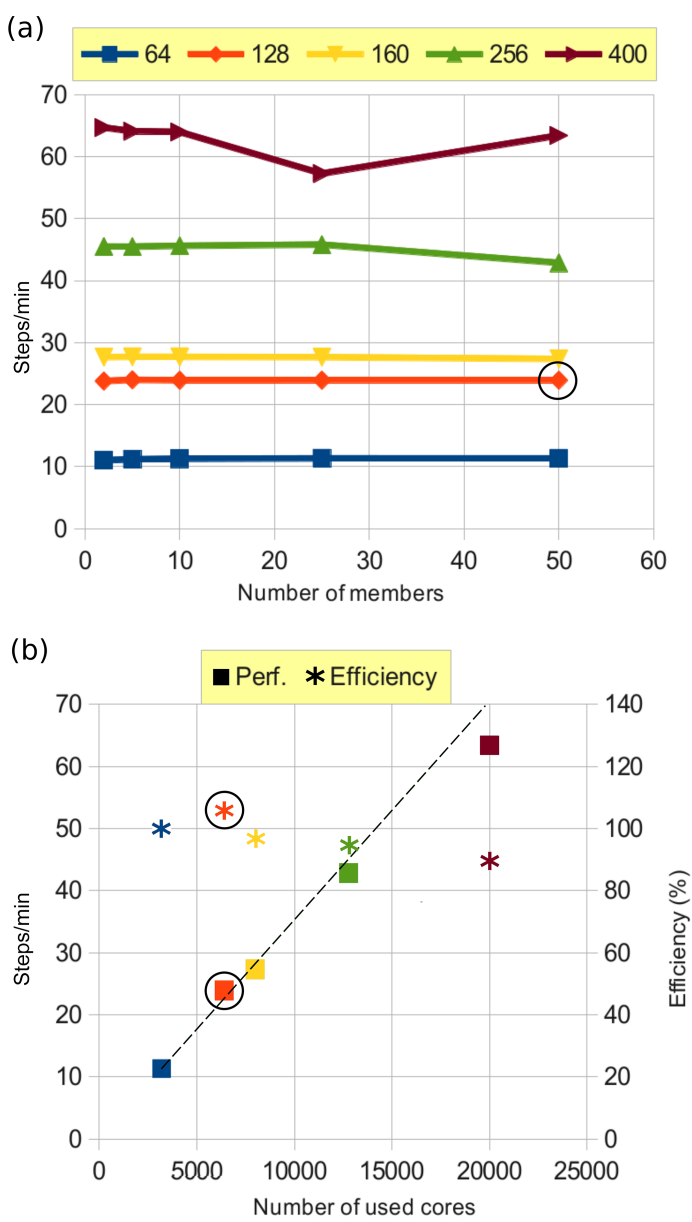

Figure 3. (a) Performance of the global ensemble configuration as a function of ensemble size $N$, for five domain decompositions: 64, 128, 160, 256, and 400 cores per member (coloured lines). (b) Performance in steps per minute and efficiency in \% of the global ensemble configuration with 50 members. The dotted line represents the theoretical speedup. The number of cores corresponds here to $N$ times the number of subdomains per member. Our final choice (50 members, 128 cores per member) is indicated with black circles.

perature given the identical atmospheric evolution that forces all members.

These temperature anomalies were computed by first removing the long-term non-linear trend of the time series derived from a local regression model (as in Grégorio et al., 2015). This detrending step acts as a non-linear high-pass temporal filter with negligible end-point effect also called local regression (LOESS) detrending (e.g. Cleveland et al., 1992; Cleveland and Loader, 1996), which successfully removes the unresolved imprints of very low-frequency variabilities (of forced or intrinsic origin), and possible nonlinear model drifts. We focus here on the ocean variability that is fully resolved in the 20-year regional simulation output; we thus choose to remove the total long-term trend of each member individually prior to plotting and analysing the ensemble statistics presented here. The mean seasonal cycle computed over the ensemble has also been removed from the monthly time series.

The ensemble-mean time series (hereafter E-mean, also noted $\widetilde{\mu_{k}}$ in Sect. 3) was then computed from these detrended time series, and illustrates the temperature evolution common to all members, i.e. forced by the atmospheric variability. The temporal standard deviation (hereafter Time-SD) of this ensemble mean thus provides an estimate of the atmospherically forced variability.

The dispersion of individual time series about the ensemble mean indicates the amount of intrinsic chaotic variability generated by the model. Its time-varying magnitude may be estimated by the ensemble standard deviation (hereafter E-SD, also noted $\widetilde{\sigma_{k}}$ in Sect. 3). Besides these low-order statistical moments, ensemble simulations actually provide an estimate of the full ensemble probability density function distribution (E-PDF) at any time, with an accuracy that increases with the number of members in the ensemble (see also Sect. 3.2).

\subsection{Initialization and evolution of the ensemble spread}

Unlike in short-range ensemble forecast exercises, we do not seek here to maximize the growth rate of the initial dispersion; we let the model feed the spread and control its evolution following its physical laws.

Figure $4 \mathrm{~b}$ and $\mathrm{d}$ confirm that the stochastic perturbation strategy (Sect. 4.2) successfully seeds an initial spread between the ensemble members. The evolution and growth rate of the temperature E-SD depend on the geographical location: it grows faster in turbulent areas such as the Gulf Stream (Fig. 4b) and slower in less-turbulent areas like the subtropical gyre (Fig. 4d). Note that the spread keeps growing after the stochastic parameterization has been switched off at the end of 1993, and tends to reach some leveled and saturated value after a few years. It is nevertheless still subject to clear modulations of its magnitude on timescales ranging from monthly to interannual. An additional 8-year experiment (not shown here) has confirmed that when the small stochastic perturbation is applied over the whole simulation instead of 1 year, the overall evolution, magnitude, and spatial patterns of E-SD, as well as the ensemble mean solution, remain unchanged. In other words, the stochastic parameterization seeds the spread during the initialization period, but the subsequent evolution and magnitude of intrinsic variability is subsequently controlled by the model non-linearities, regardless of the initial stochastic seeding.

\subsection{Spatial patterns of the ensemble spread}

Figure 5 shows maps of E-SD in the regional ensemble ENATL025, computed from annual-mean anomalies of sea surface height (SSH), sea surface temperature (SST), and temperature at 93 and $227 \mathrm{~m}$ over the last simulated year 
(a)

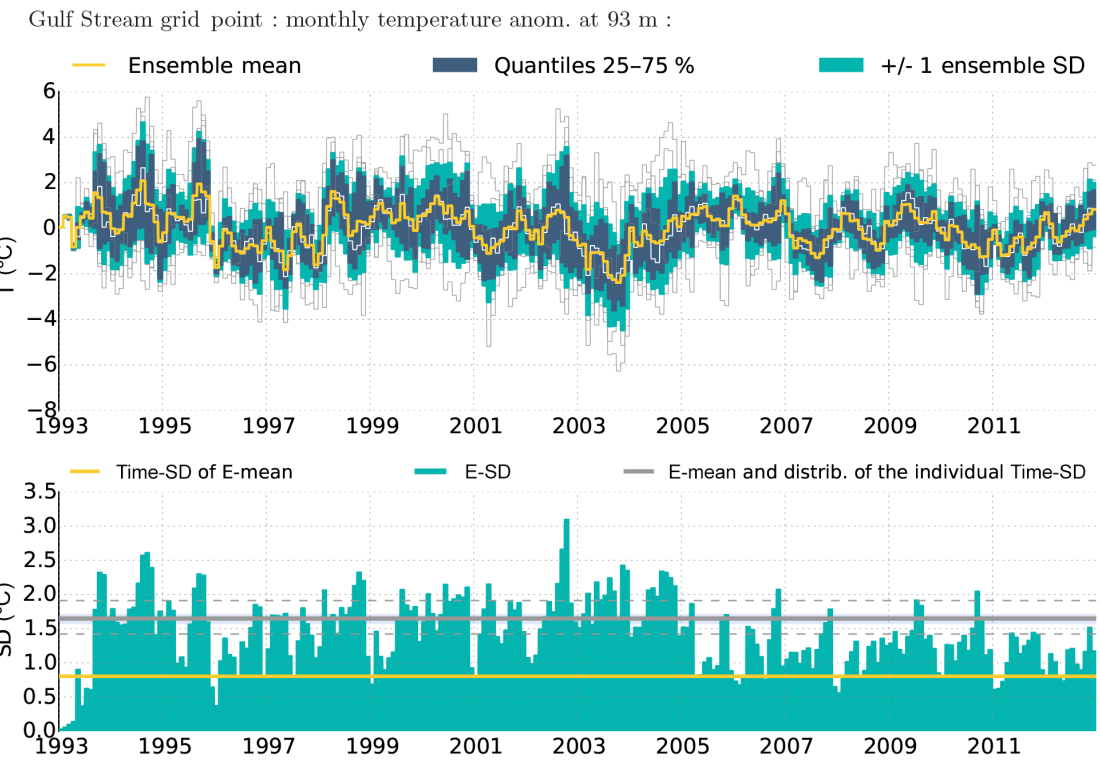

Subtropical-gyre grid point : monthly temperature anom. at $93 \mathrm{~m}$

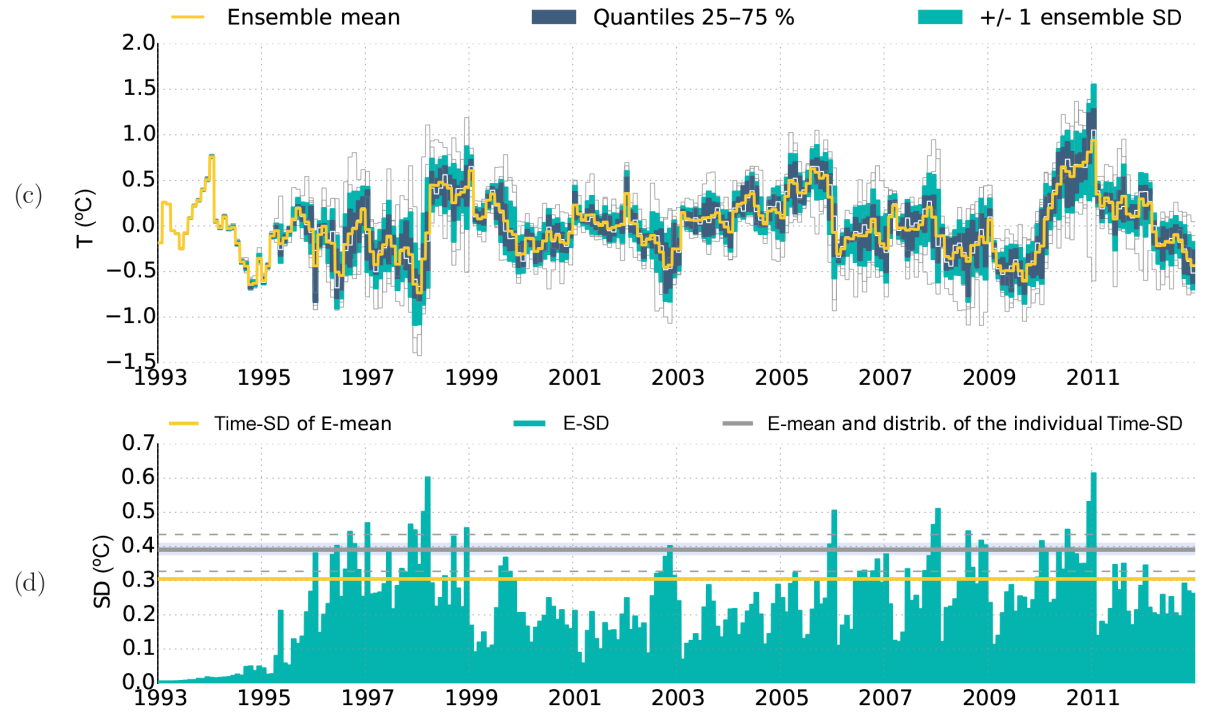

Figure 4. Ensemble statistics of the monthly temperature anomalies from the regional ensemble E-NATL025, at depth $93 \mathrm{~m}$ at two grid points: (a, b) in the Gulf Stream $\left(42^{\circ} \mathrm{N}, 56^{\circ} \mathrm{W}\right)$ and $(\mathbf{c}, \mathbf{d})$ in the North Atlantic subtropical gyre $\left(22^{\circ} \mathrm{N}, 42^{\circ} \mathrm{W}\right)$. Anomalies are shown after detrending and seasonal cycle removed (see text for details). (a) The individual trajectories with time of the 10 members appear in thin grey. E-mean is in thick yellow, the interval between quantiles Q1 $(25 \%)$ and Q3 (75\%) is filled in dark blue, and the interval E-mean \pm 1 E-SD is filled in green. (b) E-SD (intrinsic variability, green shading) is compared to the Time-SD of E-mean (forced variability, thick yellow line). Also shown in (b) is the distribution of Time-SD for the 10 members: ensemble mean of the Time-SDs (solid grey), minimum and maximum (dashed grey), and mean \pm 1 ensemble standard deviation (pale blue shading).

(i.e. 2012). These maps thus quantify the imprint of interannual intrinsic variability on these variables, and show that after 20 years of simulation, the ensemble spread has cascaded from short (mesoscale-like) periods to long timescales. Annual E-SDs reach their maxima in eddy-active regions like the Gulf Stream (Fig. 5a) and the North Equatorial Countercurrent (Fig. 5c) where hydrodynamic instabilities are strongest and continuously feed mesoscale activity (i.e. small-scale intrinsic variability), which then cascades to longer timescales. The order of magnitude of this LFIV is about $1{ }^{\circ} \mathrm{C}$ for SST and $10 \mathrm{~cm}$ for SSH in the Gulf Stream in 2012. We will compare these amplitudes to those of the atmospherically forced variability (Time-SD of E-mean) in the next section. 
(a) $2012 \mathrm{SSH}$ E-SD

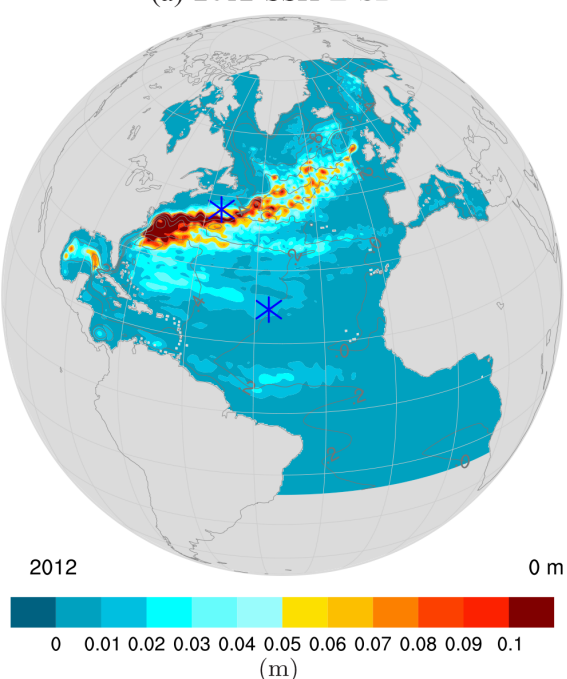

(m)

(c) 2012 T _93m E-SD

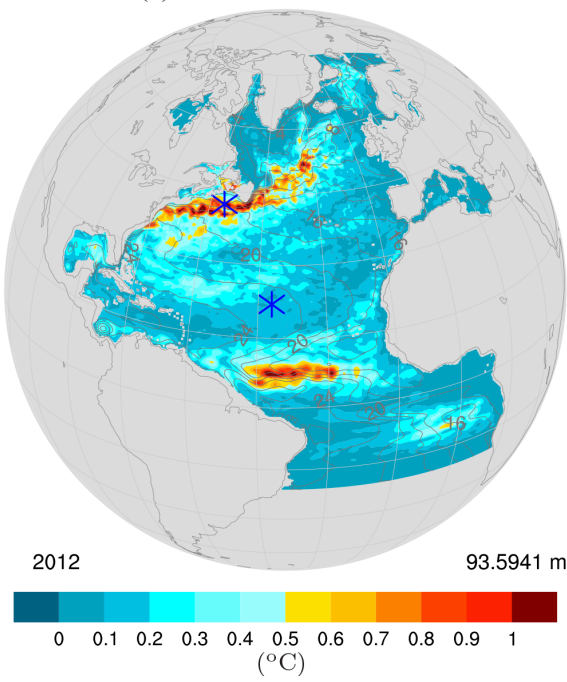

(b) 2012 SST E-SD

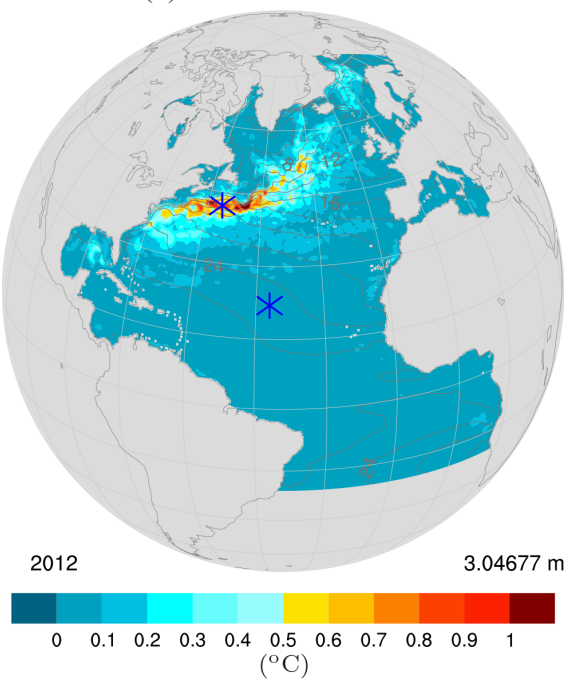

(d) $2012 \mathrm{~T} \_227 \mathrm{~m}$ E-SD

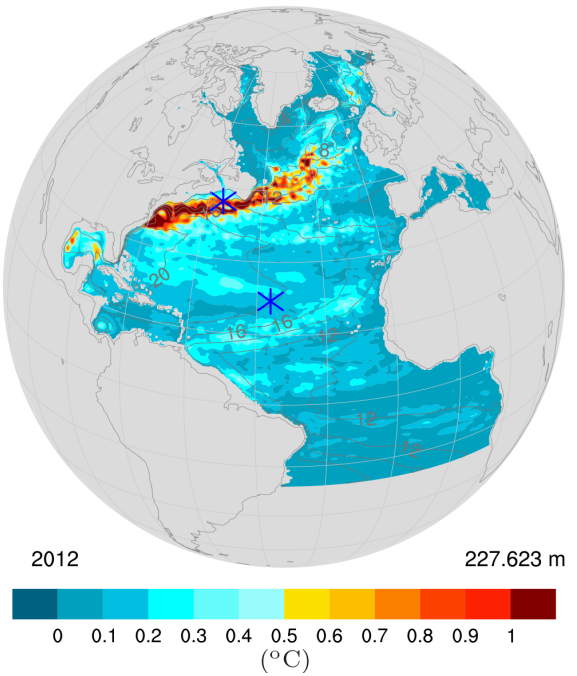

Figure 5. E-SD (shading) for year 2012 of the regional ensemble simulation E-NATL025, computed from annual means of (a) sea surface height (SSH), (b) sea surface temperature, and (c, d) temperature at depths 93 and $227 \mathrm{~m}$, respectively. The contours show the corresponding E-mean fields. The blue symbols pinpoint the two grid points at which time series are shown in Fig. 4.

Comparing Fig. 5b, c, and d also illustrates that the ensemble spread of yearly temperature (i.e. its low-frequency intrinsic variability) peaks at subsurface (around the thermocline), and tends to decrease toward the surface in eddy-quiet regions.

This is expected from the design of these ensemble simulations: each ensemble member is driven through bulk formulae by the same atmospheric forcing function, but turbulent air-sea heat fluxes differ somewhat among the ensemble because SSTs do so. This approach induces an implicit relaxation of SST toward the same equivalent air temperature (Barnier et al., 1995) within each member, hence an artificial damping of the SST spread. These experiments thus only provide a conservative estimate of the SST intrinsic variability. Note that alternative forcing strategies may alleviate or remove this damping effect: ensemble mean air-sea fluxes may be computed online at each time step and applied identically to all members (see Sect. 3.2). This alternative approach is the subject of ongoing work and will be presented in a dedicated publication.

\subsection{Magnitudes of forced and intrinsic variability}

Figure $4 \mathrm{~b}$ and $\mathrm{d}$ show how the E-SD evolves at monthly timescale, and how it compares to various Time-SDs (horizontal straight lines). The Time-SD of E-mean (thick solid 
yellow line) is a proxy for the amount of the forced variability. It turns out to be dominated by the intrinsic variability (E-SD) at the Gulf Stream grid point. In less turbulent areas like the subtropical gyre, the intrinsic variability is still about $30-50 \%$ of the forced part (Fig. 4d).

The E-SD can also be compared to the ensemble distribution of the Time-SDs of the $N$ members (see caption of Fig. 4). By construction, the Time-SD of each member is due to both the forced (shared by all members) and the intrinsic (unique to each member) variability. At the Gulf Stream grid point (Fig. 4b), these lines all lie above the Time-SD of E-mean, consistent with a high level of E-SD (i.e. intrinsic variability) contributing significantly to the total variability. At the subtropical gyre grid point, these lines fall much closer to E-mean since little intrinsic variability contributes to the total variability.

\subsection{Toward probabilistic climate diagnostics}

The variability of the atlantic meridional overturning circulation (AMOC) transport is of major influence on the climate system (e.g. Buckley and Marshall, 2016), and is being monitored at $26.5^{\circ} \mathrm{N}$ since 2004 by the RAPID array (e.g. Johns et al., 2008). These observations are shown at monthly and interannual timescales as an orange line in Fig. 6, along with their simulated counterpart from E-ORCA025. They were computed in geopotential coordinates as in Zhang (2010) and Grégorio et al. (2015), and are shown after LOESS detrending and after removing the mean seasonal cycle.

The simulated AMOC time series are in a good agreement with the observed AMOC variations at both monthly and annual timescales (Fig. 6a and c). The total (i.e. combination of forced and intrinsic) AMOC variability is computed as a Time-SD from the observed time series and from each ensemble member, and plotted in Fig. $6 \mathrm{~b}$ and $\mathrm{d}$ as gray lines. At both timescales, the total AMOC variability simulated by E-ORCA025 lies below the observed variability, consistent with the fact that the model seems to miss a few observed peaks (e.g. 2005, 2009, and 2013 on the annual time series). Figure $6 \mathrm{~b}$ and $\mathrm{d}$ also highlight the substantial imprint of chaotic intrinsic variability on this climate-relevant oceanic index at both timescales: at interannual timescale, the AMOC intrinsic variability is weaker than the forced variability, but amounts to about $30 \%$ of the latter. A more in-depth investigation of the relative proportion of intrinsic and forced variability in the AMOC and of the variations of the intrinsic contribution with time is currently underway and will be the subject of a dedicated publication.

\section{Conclusions}

We have presented in this paper the technical implementation of a new, probabilistic version of the NEMO ocean modelling system. Ensemble simulations with $N$ members run- ning simultaneously in a single NEMO executable are made possible through a double MPI parallelization strategy acting both in the spatial and the ensemble dimensions (Fig. 2), and an optimized dimensioning and implementation of the $\mathrm{I} / \mathrm{O}$ servers (XIOS) on the computing nodes.

The OCCIPUT project was presented here as an example application of these new modelling developments. Its scientific focus is on studying and comparing the intrinsic/chaotic and the atmospherically forced parts of the ocean variability at monthly to multidecadal timescales (e.g. Penduff et al., 2014). For this purpose, we have performed a large ensemble of 50 global ocean-sea-ice hindcasts over the period 19602015 at $1 / 4^{\circ}$ resolution, and a reduced-size North Atlantic regional ensemble. These experiments simultaneously simulate the forced and chaotic variabilities, which may then be diagnosed via the ensemble mean and ensemble standard deviation, respectively. The global OCCIPUT ensemble simulation was achieved in a total of 19 million CPU hours on the PRACE French Tier-0 Curie supercomputer, supported by a PRACE grant. It produced about $100 \mathrm{~TB}$ of archived outputs.

The members are all driven by the same realistic atmospheric boundary conditions (DFS5.2) through bulk formulae, and represent $N$ independent realizations of the same oceanic hindcast. The ensemble experiments performed here have validated our experimental strategy: a stochastic parameterization was activated for 1 year to trigger the growth of the ensemble spread (see Sects. 3.3 and 4.2); the subsequent growth and saturation of the spread is then controlled by the model nonlinearities. Our results also confirm that the spread cascades from short and small (mesoscale) scales to large and long scales. The imprint of intrinsic chaotic variability on various indices turns out to be large, including at large spatial scales and timescales: the AMOC chaotic variability represents about $30 \%$ of the atmospherically forced variability at interannual timescale. These preliminary results illustrate the importance of this low-frequency oceanic chaos, and advocate for the use of such probabilistic modelling approaches for oceanic simulations driven by a realistic timevarying atmospheric forcing. This approach brings, in particular, new insights on the imprint of this low-frequency chaos on climate-related oceanic indices, and thus helps anticipate the behaviour of the next generation of coupled climate models that will incorporate eddying-ocean components. Ongoing investigations focus on these questions and will be the subject of dedicated papers.

Our probabilistic NEMO version includes several new features. The generic stochastic parameterization, used here on the equation of state to trigger the growth of the ensemble spread, can be applied to other parameters to simulate model or subgrid-scale uncertainties. The MPI communication between members allows the online computation of ensemble statistics (PDFs, variances, covariances, quantiles, etc.) across the ensemble members, which may be saved at any frequency and location and for any variable thanks to the flexible XIOS servers. 
(a)

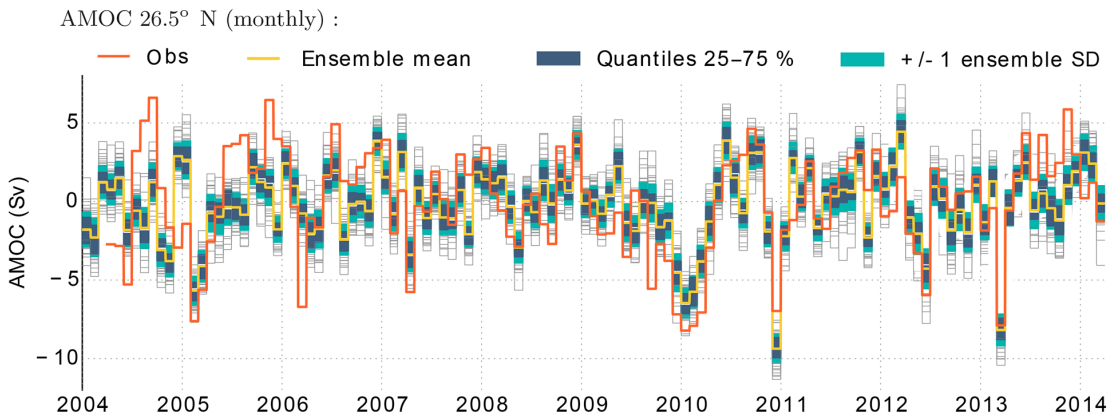

(b)

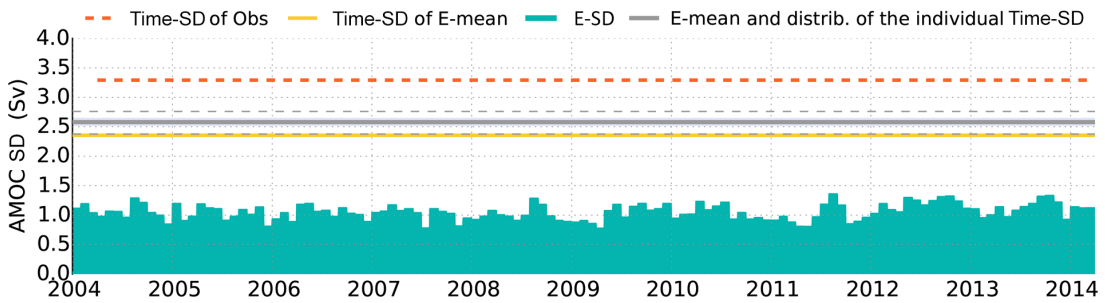

(c)

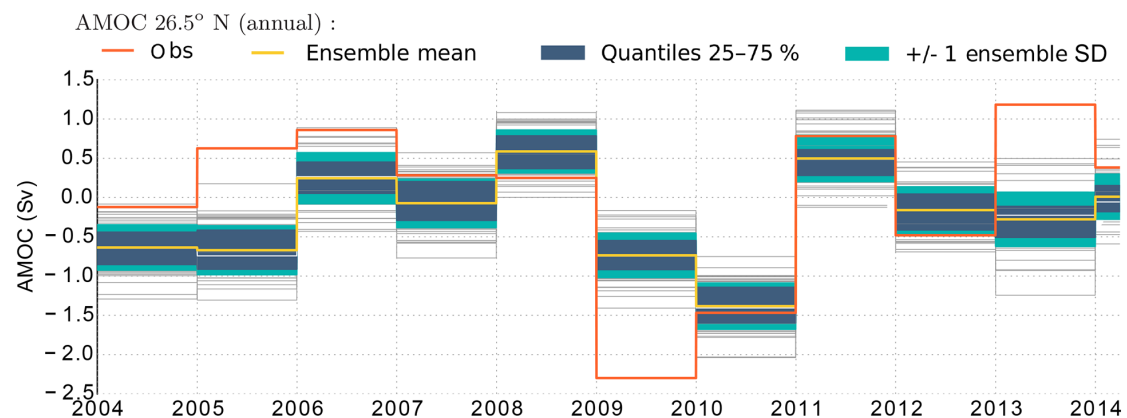

(d)

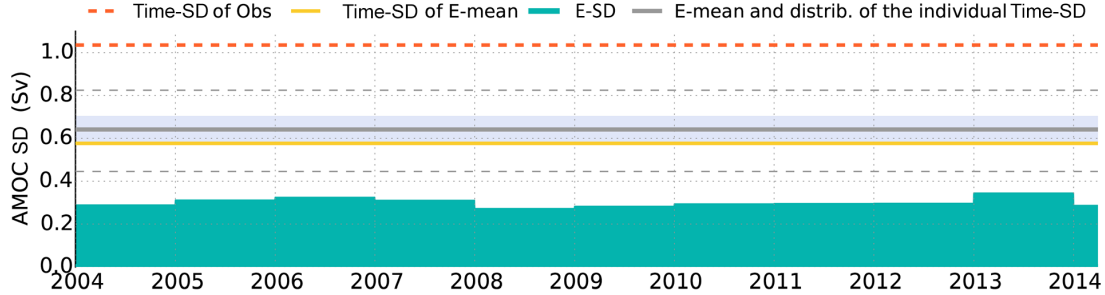

Figure 6. Same as Fig. 4 but for AMOC anomalies at $26^{\circ} \mathrm{N}$ in the global ensemble E-ORCA025, from (a, b) monthly and (c, d) annual means. In addition, AMOC observational estimates from RAPID at $26^{\circ} \mathrm{N}$ are shown in orange (see text for details).

The size $N$ of the ensemble simulation depends on the objectives of the study, the desired accuracy of ensemble statistics, and the available computing resources. Our choice $N=50$ allows a good accuracy $(1 / \sqrt{50}= \pm 14 \%)$ for estimating the ensemble means and standard deviations. Moreover, this choice allows the estimation of ensemble deciles (with five members per bin) for the detection of possibly bimodal or other non-gaussian features of ensemble PDFs; such behaviours were indeed detected in simplified ensemble experiments (e.g. Pierini, 2014) and may appear in ours. Given our preliminary tests with E-NATL025, $N=50$ appeared as a satisfactory compromise between our need for a long global $1 / 4^{\circ}$ simultaneous integration, our scientific objectives, PRACE rules (expected allocation and elapsed time, jobs' duration, etc), and Curie's technical features (processor performances, memory, communication cost). Our tests also indicate that the convergence of ensemble statistics with $N$ depends on the variables, metrics and regions under consideration. For all these reasons, $N$ must be chosen adequately for each study.

More generally, this numerical system computes the temporal evolution of the full PDF of the 3-D, multivariate states of the ocean and sea ice. A very interesting perspective is the online use of the PDF of any state variable or derived 
quantity (or other statistics such as ensemble means, variances, covariances, skewnesses, etc.) for the computation of the next time step during the integration. This would allow, for instance, distinct treatments of the ensemble mean (forced variability) or the ensemble spread (intrinsic variability) during the integration, e.g. for data assimilation purposes. This NEMO version can therefore solve the oceanic Fokker-Planck equation, which may open new avenues in term of experimental design for operational, climate-related, or process-oriented oceanography.

\section{Code availability}

The ensemble simulations described in this paper have been performed using a probabilistic ocean modelling system based on NEMO 3.5. The model code for NEMO 3.5 is available from the NEMO website (www.nemo-ocean.eu). On registering, individuals can access the code using the open-source subversion software (http://subversion.apache. org). The revision number of the base NEMO code used for this paper is 4521 . The probabilistic ocean modelling system is fully available from the Zenodo website (https: //zenodo.org/record/61611) with doi:10.5281/zenodo.61611. The authors warn that this provision of sources does not imply warranties and support; they decline any responsibility for problems, errors, or incorrect usage of NEMO. Additional information can be found on the NEMO website.

The ensemblist features of the model are based on a generic tool implemented in the NEMO parallelization module.

The computer code includes one new FORTRAN routine (mpp_ens_set; see Algorithm 1) which defines the MPI communicators required to perform simultaneous simulations, and to compute online ensemble diagnostics. This routine returns to each NEMO instance: (i) the MPI communicator that it must use to run the model, and (ii) the index of the ensemble member to be run. This index can then be used by NEMO to modify (i) the input filenames (initial condition, forcing, parameters), (ii) the output filenames (model state, restart file, diagnostics), and (iii) the seed of the random number generator used in the stochastic parameterizations.

The online computation of ensemble diagnostics requires additional routines, for instance to compute the ensemble mean or standard deviation of model variables (mpp_ens_ave_std, see Algorithm 2). This routine uses the diagnostic communicators defined by mpp_ens_set to perform summations over all ensemble members.

As can be seen from these routines, this implementation is generic and can be implemented in any kind of model that is already parallelized using a domain decomposition method.
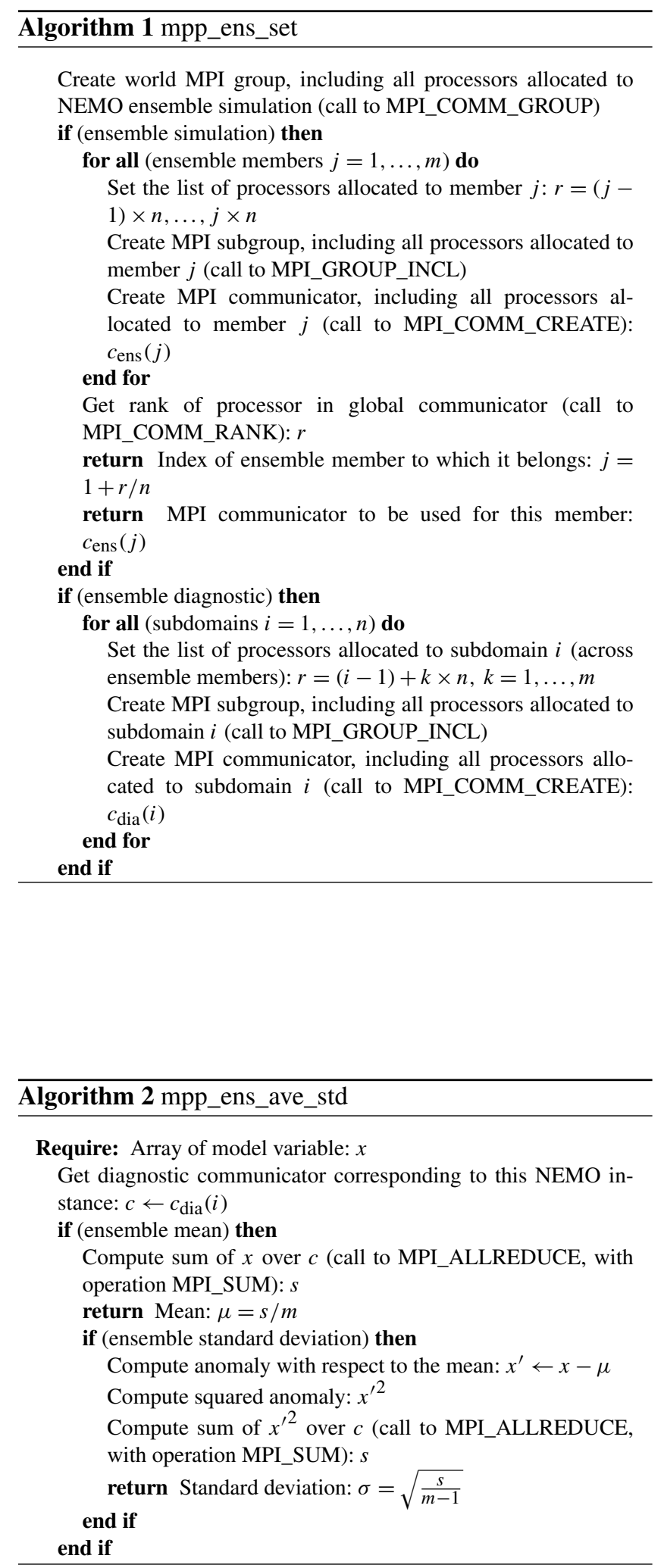
Competing interests. The authors declare that they have no conflict of interest.

Acknowledgements. This work is mainly a contribution to the OCCIPUT project, which is supported by the Agence Nationale de la Recherche (ANR) through contract ANR-13-BS06-0007-01. We acknowledge that the results of this research have been achieved using the PRACE Research Infrastructure resource Curie based in France at TGCC. The support of the TGCC-CCRT hotline from CEA, France, to the technical work is gratefully acknowledged. Some of the computations presented in this study were performed at TGCC under allocations granted by GENCI. This work also benefited from many interactions with the DRAKKAR ocean-modelling consortium, with the SANGOMA and CHAOCEAN projects. DRAKKAR is the International Coordination Network (GDRI) established between the Centre National de la Recherche Scientifique (CNRS), the National Oceanography Centre in Southampton (NOCS), GEOMAR in Kiel, and IFREMER. SANGOMA is funded by the European Community's Seventh Framework Programme FP7/2007-2013 under grant agreement 283580. CHAOCEAN is funded by the Centre National d'études Spatiales (CNES) through the Ocean Surface Topography Science Team (OST/ST). The authors are grateful for useful comments from three anonymous reviewers; they also thank the NEMO System Team and Yann Meurdesoif for interesting discussions about the development of the probabilistic version of NEMO. Laurent Bessières and Stéphanie Leroux are supported by ANR. Jean-Michel Brankart, Jean-Marc Molines, Pierre-Antoine Bouttier, Thierry Penduff, and Bernard Barnier are supported by CNRS. Marie-Pierre Moine and Laurent Terray are supported by CERFACS, and Guillaume Sérazin by CNES and Région Midi-Pyrénées.

Edited by: David Ham

Reviewed by: three anonymous referees

\section{References}

Barnier, B., Siefridt, L., and Marchesiello, P.: Thermal forcing for a global ocean circulation model using a three-year climatology of ECMWF analyses, J. Mar. Syst., 6, 363-380, doi:10.1016/09247963(94)00034-9, 1995.

Barnier, B., Madec, G., Penduff, T., Molines, J.-M., Treguier, A.M., Le Sommer, J., Beckmann, A., Biastoch, A., Böning, C., Dengg, J., Derval, C., Durand, E., Gulev, S., Remy, E., Talandier, C., Theetten, S., Maltrud, M., McClean, J., and De Cuevas, B.: Impact of partial steps and momentum advection schemes in a global ocean circulation model at eddy permitting resolution, Ocean Dynam., 56, 543-567, 2006.

Bertino, L., Evensen, G., and Wackernagel, H.: Sequential data assimilation techniques in oceanography, Int. Stat. Rev., 71, 223 241, 2003.

Brankart, J.-M.: Impact of uncertainties in the horizontal density gradient upon low resolution global ocean modelling, Ocean Model., 66, 64-76, 2013.

Brankart, J.-M., Testut, C.-E., Béal, D., Doron, M., Fontana, C., Meinvielle, M., Brasseur, P., and Verron, J.: Towards an improved description of ocean uncertainties: effect of local anamorphic transformations on spatial correlations, Ocean Sci., 8, 121-142, doi:10.5194/os-8-121-2012, 2012.

Brankart, J.-M., Candille, G., Garnier, F., Calone, C., Melet, A., Bouttier, P.-A., Brasseur, P., and Verron, J.: A generic approach to explicit simulation of uncertainty in the NEMO ocean model, Geosci. Model Dev., 8, 1285-1297, doi:10.5194/gmd-8-12852015, 2015.

Buckley, M. W. and Marshall, J.: Observations, inferences, and mechanisms of Atlantic Meridional Overturning Circulation variability: A review, Rev. Geophys., 54, 5-63, doi:10.1002/2015RG000493, 2016.

Candille, G. and Talagrand, O.: Evaluation of probabilistic prediction systems for a scalar variable, Q. J. Roy. Meteor. Soc., 131, 2131-2150, 2005.

Candille, G., Brankart, J.-M., and Brasseur, P.: Assessment of an ensemble system that assimilates Jason-1/Envisat altimeter data in a probabilistic model of the North Atlantic ocean circulation, Ocean Sci., 11, 425-438, doi:10.5194/os-11-425-2015, 2015.

Cleveland, W. S., Grosse, E., Shyu, M. J., Grosse, E., Shyu, M. J., and Shyu, M. J.: A package of $\mathrm{C}$ and fortran routines for fitting local regression models, in: Statistical methods, edited by: Chambers, J. M., S. Chapman and Hall Ltd., London, UK, 1992.

Cleveland, W. S. and Loader, C.: Smoothing by local regression: Principles and methods, in: Statistical theory and computational aspects of smoothing, Springer, 10-49, 1996.

Combes, V. and Lorenzo, E.: Intrinsic and forced interannual variability of the Gulf of Alaska mesoscale circulation, Prog. Oceanogr., 75, 266-286, 2007.

Deser, C., Terray, L., and Phillips, A. S.: Forced and internal components of winter air temperature trends over North America during the past 50 years: Mechanisms and implications, J. Climate, 29, 2237-2258, 2016.

Dussin, R., Barnier, B., Brodeau, L., and Molines, J.-M.: The making of Drakkar forcing set DFS5, DRAKKAR/MyOcean Report 01-04-16, LGGE, Grenoble, France, 2016.

Evensen, G.: Sequential data assimilation with a nonlinear quasigeostrophic model using monte carlo methods to forecast error statistics, J. Geophys. Res.-Oceans, 99, 10143-10162, 1994.

Grégorio, S., Penduff, T., Sérazin, G., Molines, J. M., Barnier, B., and Hirshi, J.: Intrinsic variability of the Atlantic meridional overturning circulation at interannual-to- multidecadal timescales, J. Phys. Oceanogr., 45, 1929-1946, doi:10.1175/JPO-D-14-0163.1, 2015.

Haarsma, R. J., Roberts, M. J., Vidale, P. L., Senior, C. A., Bellucci, A., Bao, Q., Chang, P., Corti, S., Fuckar, N. S., Guemas, V., von Hardenberg, J., Hazeleger, W., Kodama, C., Koenigk, T., Leung, L. R., Lu, J., Luo, J.-J., Mao, J., Mizielinski, M. S., Mizuta, R., Nobre, P., Satoh, M., Scoccimarro, E., Semmler, T., Small, J., and von Storch, J.-S.: High Resolution Model Intercomparison Project (HighResMIP v1.0) for CMIP6, Geosci. Model Dev., 9, 4185-4208, doi:10.5194/gmd-9-4185-2016, 2016.

Hirschi, J. J.-M., Blaker, A. T., Sinha, B., Coward, A., de Cuevas, B., Alderson, S., and Madec, G.: Chaotic variability of the meridional overturning circulation on subannual to interannual timescales, Ocean Sci., 9, 805-823, doi:10.5194/os-9-805-2013, 2013.

Johns, W., Beal, L., Baringer, M., Molina, J., Cunningham, S., Kanzow, T., and Rayner, D.:Variability of shallow and deep western 
boundary currents off the bahamas during 2004-05: Results from the $26 n$ rapid-moc array, J. Phys. Oceanogr., 38, 605-623, 2008.

Kay, J. E., Deser, C., Phillips, A., Mai, A., Hannay, C., Strand, G., Arblaster, J., Bates, S., Danabasoglu, G., Edwards, J., Holland, M., Kushner, P., Lamarque, J.-F., Lawrence, D., Lindsay, K., Middleton, A., Munoz, E., Neale, R., Oleson, K., Polvani, L., and Vertenstein, M.: The Community Earth System Model (CESM) Large Ensemble Project: A community resource for studying climate change in the presence of internal climate variability, B. Am. Meteorol. Soc., 96, 1333-1349, 2015.

Lermusiaux, P. F.: Uncertainty estimation and prediction for interdisciplinary ocean dynamics, J. Comput. Phys., 217, 176-199, 2006.

Madec, G.: Nemo ocean engine, Tech. rep., NEMO team, 2012.

Palmer, T. N.: Predictability of weather and climate, Cambridge University Press, 2006.

Penduff, T., Juza, M., Barnier, B., Zika, J., Dewar, W. K., Treguier, A.-M., Molines, J.-M., and Audiffren, N.: Sea level expression of intrinsic and forced ocean variabilities at interannual time scales, J. Climate, 24, 5652-5670, 2011.

Penduff, T., Barnier, B., Terray, L., Bessières, L., Sérazin, G., Gregorio, S., Brankart, J., Moine, M., Molines, J., and Brasseur, P.: Ensembles of eddying ocean simulations for climate, CLIVAR Exchanges, Special Issue on High Resolution Ocean Climate Modelling, 19, 2014.
Pierini, S.: Ensemble Simulations and Pullback Attractors of a Periodically Forced Double-Gyre System, J. Phys. Oceanogr., 44, 3245-3254, 2014.

Sérazin, G., Penduff, T., Grégorio, S., Barnier, B., Molines, J. M., and Terray, L.: Intrinsic variability of sea-level from global $1 / 12^{\circ}$ ocean simulations: spatio-temporal scales, J. Climate, 28, 42794292, 2015.

Toth, Z., Talagrand, O., Candille, G., and Zhu, Y.: Probability and Ensemble Forecasts, in: Forecast Verification: A Practitioners Guide in Atmospheric Science, Wiley, UK, 137-163, ISBN: 0471-49 759-2, 2003.

Ubelmann, C., Verron, J., Brankart, J., Cosme, E., and Brasseur, P.: Impact of data from upcoming altimetric missions on the prediction of the three-dimensional circulation in the tropical atlantic ocean, J. Oper. Oceanogr., 2, 3-14, 2009.

Van Leeuwen, P. J.: Particle filtering in geophysical systems, Mon. Weather Rev., 137, 4089-4114, 2009.

Zhang, R.: Latitudinal dependence of Atlantic meridional overturning circulation (AMOC) variations, Geophys. Res. Lett., 37, L16703, doi:10.1029/2010GL044474, 2010. 